\title{
Linearly Stable Orbits in 3 Dimensional Billiards*
}

\author{
Maciej P. Wojtkowski \\ Department of Mathematics, University of Arizona, Tucson AZ 85721, USA
}

\begin{abstract}
We construct linearly stable periodic orbits in a class of billiard systems in 3 dimensional domains with boundaries containing semispheres arbitrarily far apart. It shows that the results about planar billiard systems in domains with convex boundaries which have nonvanishing Lyapunov exponents cannot be easily extended to 3 dimensions.
\end{abstract}

\section{Introduction}

Since the work of Sinai [S] we know that dispersing billiards (billiard systems in domains with concave boundaries) have strong mixing properties in all of the phase space. Conceptually such systems are close to geodesic flows on manifolds of nonpositive sectional curvature. In particular the dimension of the domain does not affect the basic features of the dynamics [C-S]. Bunimovich [B1] discovered that convex pieces of the boundary may produce the same scattering effect in planar billiards. He constructed examples of convex planar domains built of arcs of circles and straight lines (the stadium) in which billiard systems have the hyperbolic behavior. In [W1] the rigid requirement of constant curvature on the convex pieces of the boundary was replaced by the condition that $r^{\prime \prime}(s) \leqq 0$, where $r(s)$ is the radius of curvature as a function of arc length. Subsequently Markarian [M] and Donnay [D] showed that other conditions may suffice. It was already clear in [W1] that putting two convex pieces of the boundary sufficiently far apart makes the orbits that go back and forth between the two completely unstable (the difficulty there is to include the "glancing" pieces of the orbit: many consecutive reflections at large angles in one convex piece of the boundary). This idea is further supported by the work of Donnay, who shows that basically arbitrary sufficiently small perturbations of the stadium have nonzero Lyapunov exponents. (Bunimovich [B2] made some vague claims along these lines before but so far he has not published the details.) The natural question arises: do these planar (locally) convex domains have higher dimensional counterparts? More specifically we can ask if

\footnotetext{
* Supported in part by the NSF Grant DMS-8807077 and the Sloan Foundation
} 
putting two convex pieces of the boundary sufficiently far apart makes all the periodic orbits held by them completely unstable (no Floquet exponents on the unit circle).

In the present paper we make a contribution towards the negative answer to these questions. We construct a linearly stable periodic billiard orbit which reflects only in eight semispheres in $\mathbf{R}^{3}$. Given any distance $l$ (not smaller than the diameter of the semispheres) we consider two parallel planes at this distance. We attach four semispheres to each of the planes (on the outside). The positions of the semispheres are the free parameters in our construction. We adjust them in such a way that there is a highly symmetric periodic billiard orbit which reflects only in the semispheres. We are still left with some free parameters and we show that in a certain range of these parameters the periodic orbit is linearly stable.

There are enough parameters in this construction to escape any resonances in the Floquet exponents. One can naturally expect that also the Birkhoff normal form is typically nondegenerate for this periodic orbit so that by the KAM theory [A] there is a subset of positive Lebesgue measure in a neighborhood of the orbit which carries quasiperiodic motions. Because of the lack of working criteria for nondegeneracy of the Birkhoff normal form we were unable to complete this last step. The straightforward computation of the Birkhoff normal form is in our case out of the question (the orbit has at least 32 reflections in the semispheres).

Our construction shows that even with the simplest convex surface - the sphere, the presence of linearly stable orbits cannot be excluded by merely putting the convex pieces sufficiently far apart. In a recently published paper Bunimovich [B3] claims that billiard systems in several multidimensional domains with convex boundaries have strong mixing properties in all of the phase space. In view of our construction we have doubts whether this can be indeed proven for all of his examples (we believe that the long linearly stable periodic orbits are an exception rather than a rule but we doubt that their absence can be secured by simple conditions). As a byproduct of our discussion one case of scattering behavior emerges - the reflections in a small piece of a sphere. It was already known to Bunimovich, [B3, B4].

We would like to note that Knauf [K], and Donnay and Liverani [D-L] found large classes of finite range potentials which produce nonvanishing Lyapunov exponents in all of the phase space of a particle moving in the planar central field. Yet they were unable to extend it to three (or more) dimensions. We think that similar mechanisms are at work there.

The plan of the paper is as following. In Sect. 1 we discuss the linearized dynamics around a billiard orbit (the evolution of orthogonal Jacobi fields). We write down the reflection formulas for a sphere and we point out to their features crucial to the subsequent construction. Section 2 contains the geometric details of the construction. The analytic details of the proof that the orbit is linearly stable in some range of the parameters can be found in Sect. 3.

\section{Description of the Linearized Dynamics}

A billiard system can be viewed as a kind of geodesic flow so that it is natural to introduce Jacobi fields (infinitesimal variations) for a billiard orbit. In particular 
we describe the linearized dynamics around a billiard orbit by orthogonal Jacobi fields.

We will consider billiard systems only in domains in $\mathbf{R}^{3}$. An orthogonal Jacobi field along a piece of an orbit between reflections at the boundary is a vector field $\xi(t) \in V$ such that

$$
\xi^{\prime \prime}=0
$$

and $V$ is the plane perpendicular to the orbit (we identify by parallel translation the planes perpendicular to the orbit at different points, not separated by a reflection at the boundary).

We need also the reflection formulas which describe what happens to a Jacobi field at the moment of reflection. Since we will consider only billiard orbits reflecting on the inside of spheres we give the reflection formulas for that case alone. Let $\mathscr{S}$ be (a piece of) a sphere of radius $r$ and $p \in \mathscr{S}$ be the point of reflection which takes place on the inside of $\mathscr{S}$. Let further $\mathbf{n}$ be the inside unit normal vector at $p, \mathbf{w}^{-}$ be the unit direction vector of the incoming billiard orbit and $\mathbf{w}^{+}=\mathbf{w}^{-}-2\left\langle\mathbf{n}, \mathbf{w}^{-}\right\rangle \mathbf{n}$ the unit direction vector of the outgoing billiard orbit. We identify the planes $V^{-}$ and $V^{+}$perpendicular to $\mathbf{w}^{-}$and $\mathbf{w}^{+}$respectively by reflection in the tangent plane at $p$. Let $\varphi, 0 \leqq \varphi \leqq \pi / 2$, be the angle of incidence, i.e. $\cos \varphi=\left\langle\mathbf{n}, \mathbf{w}^{+}\right\rangle$. We assume that $0<\varphi<\pi / 2$ so that there is the distinguished plane $W$ to which the orbit is parallel, $W$ is spanned by $\mathbf{w}^{-}$and $\mathbf{w}^{+}$. If we have several consecutive reflections in the same sphere then all the segments of the orbit are parallel to $W$. We have further that $V=V^{-}=V^{+}$splits naturally into $V=V_{p} \oplus V_{t}$, where $V_{p}=V \cap W$ and $V_{t}$ is the orthogonal complement of $V_{p}$ in $V$.

An orthogonal Jacobi field at the moment prior to the reflection is given by $(\xi, \eta) \in V \times V$, where $\eta=\xi^{\prime}$ and at the moment just after the reflection by $(\tilde{\xi}, \tilde{\eta}) \in V \times V$. The relation of $(\tilde{\xi}, \tilde{\eta})$ and $(\xi, \eta)$ is given by a linear operator on $V \times V=\left(V_{p} \times V_{p}\right) \oplus$ $\left(V_{t} \times V_{t}\right)$, which in this representation of $V \times V$ has the following block diagonal form:

$$
\left[\begin{array}{cccc}
1 & 0 & & \\
-\frac{2}{r \cos \varphi} & 1 & & \\
& & 1 & 0 \\
& & -\frac{2 \cos \varphi}{r} & 1
\end{array}\right]
$$

This formula follows immediately from the general reflection formula, see for example [W2], Appendix B.

The matrix (2) deserves special attention. Let us note that the first $2 \times 2$ block describes the evolution of orbit variations (Jacobi fields) in the plane of the orbit $W$, while the second block describes the evolution of orbit variations orthogonal to $W$. In the following we will be referring to planar Jacobi fields (variations) - in $V_{p}$ and transversal Jacobi fields (variations) - in $V_{t}$. We can see that for $\varphi$ close to $\pi / 2$ the effect of the reflection on planar and transversal variations is drastically 
different: the transversal Jacobi field changes like the planar in a reflection at a sphere with much larger radius. We know from the study of planar convex billiards that if the boundary of the billiard table has the range of curvature all the way to zero we may expect stable periodic orbits. At least all the known examples of strongly mixing billiard systems in (locally) convex planar domains have all convex pieces of the boundary with curvature bounded away from zero. Thus it appears that passing to the 3 dimensional space we encounter difficulties comparable to the smoothening of the Bunimovich stadium already when we consider reflections in a sphere.

Suppose now that our billiard orbit experiences several consecutive reflections in the same sphere, more precisely we consider the complete passage through a semisphere. Our orbit lies in one plane passing through the center of the sphere. We want to derive formulas connecting Jacobi fields at the entrance to the semisphere with Jacobi fields at the exist. For planar Jacobi fields the operator in $V_{p} \times V_{p}$ which describes the evolution from $p_{0}$ to $\tilde{p}_{0}$; see Fig. 1 , is given by the matrix

$$
(-1)^{n}\left[\begin{array}{cc}
1 & 0 \\
\frac{2 n}{r \cos \varphi} & 1
\end{array}\right],
$$

where $n$ is the number of reflections in the semisphere.

For transversal Jacobi fields we choose to describe the evolution from point $p_{1}$ to $\tilde{p}_{1}$, see Fig. 1, and we get the following operator on $V_{t} \times V_{t}$

$$
\left[\begin{array}{cc}
-\frac{\cos \beta}{\cos \alpha} & 0 \\
\frac{\sin (\alpha+\beta)}{r \sin \varphi} & -\frac{\cos \alpha}{\cos \beta}
\end{array}\right] .
$$

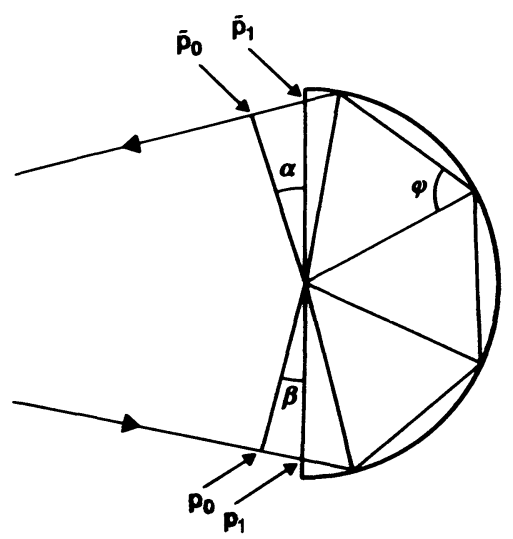

Fig. 1. 
The connection between $\alpha, \beta, \varphi$ and the number of reflections $n$ is given by

$$
(n-1) \pi=2 n \varphi+\alpha+\beta .
$$

In the choice of points $p_{0}, p_{1}, \tilde{p}_{0}$ and $\tilde{p}_{1}$ we aimed at making the resulting matrices triangular. The matrices (3) and (4) were obtained from (1) and (2) by straightforward matrix multiplication and a bit of high school geometry.

We can see that for the evolution of transversal Jacobi fields by (4) there are two distinct cases: converging, $\alpha+\beta>0$, and diverging, $\alpha+\beta<0$ (when $\alpha+\beta>0$ the outgoing ray intersects the incoming ray and when $\alpha+\beta<0$ it does not - the two diverge). In the diverging case all the nonzero entries of the matrix (4) have the same sign which is also always true for the matrix (3). It leads to the following phenomenon: if we consider a two parameter infinitesimal family of billiard orbits perpendicular to a convex surface, i.e. diverging, then after passing through the semisphere the family emerges as a diverging family (the orbits are again perpendicular to a convex surface). This makes the net effect of passing through a semisphere similar to a reflection in a concave surface (like on the outside of a sphere). Billiard systems with concave boundaries (dispersing) cannot hold a linearly stable periodic orbit (see for example [W2]). It is clear that if we replace the semisphere with a small piece of the sphere (the angle at which we see it from the center less than $\pi / 2$ ) then only the diverging case is possible. This fact was already known to Bunimovich [B4].

We will construct a linearly stable periodic billiard orbit held by eight semispheres where

1. all the passages through the semispheres are of converging type,

2. the planar and transversal variations get interchanged on the way between some semispheres.

These are the two basic elements in our construction.

\section{Construction of the Periodic Orbit}

Our periodic orbit lives on the four side faces of the rectangular box in Fig. 2. In particular the bold edges in Fig. 2 are part of the orbit. The length of the box

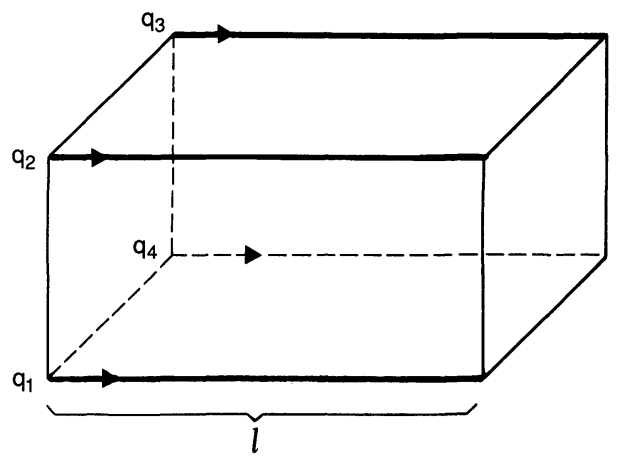

Fig. 2. 


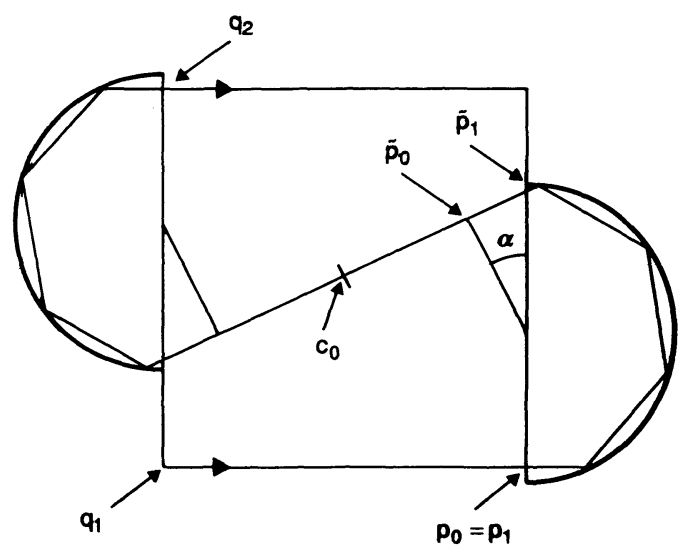

Fig. 3.

is an arbitrary given $l$, it can be arbitrarily large but should not be too small; the exact condition will be formulated later on. Figure 3 shows how the orbit gets from $q_{1}$ to $q_{2}$ by the way of $n$ reflections in each of two semispheres attached at the sides of the box. Then in the same fashion reflections in another two spheres attached similarly take the orbit from $q_{2}$ to $q_{3}$, etc., until it comes back to $q_{1}$. We need eight spheres altogether. We will take care that they do not intersect.

The configuration in Fig. 3 is centrally symmetric about the center $c_{0}$. Let us summarize some of the geometric quantities that play a role in the computations $(d(\cdot, \cdot)$ stands for the distance of two points):

$$
\begin{aligned}
d\left(\tilde{p}_{0}, \tilde{p}_{1}\right) & =r \sin \varphi \tan \alpha, \\
d\left(c_{0}, \tilde{p}_{1}\right) & =\frac{l}{2 \cos \alpha}, \\
\tilde{l}:=2 d\left(c_{0}, \tilde{p}_{0}\right) & =\frac{l}{\cos \varphi}-2 r \sin \varphi \tan \alpha, \\
d\left(q_{1}, q_{2}\right) & =2 r \sin \varphi\left(1+\frac{1}{\cos \alpha}\right)-l \tan \alpha .
\end{aligned}
$$

In this construction there is so far one continuous parameter $-\alpha$ (or $\varphi$ ) and one discrete parameter - the number $n$ of reflections in the sphere. We have that

$$
0 \leqq \alpha<\frac{\pi}{2 n-1}
$$

The evolution of orthogonal Jacobi fields on the way from $q_{1}$ to $q_{2}$ is described by the following matrix products. For planar Jacobi fields

$$
\mathscr{P}=\left[\begin{array}{cc}
1 & 0 \\
\frac{2 n}{r \cos \varphi} & 1
\end{array}\right]\left[\begin{array}{ll}
1 & \tilde{l} \\
0 & 1
\end{array}\right]\left[\begin{array}{cc}
1 & 0 \\
\frac{2 n}{r \cos \varphi} & 1
\end{array}\right]\left[\begin{array}{ll}
1 & l \\
0 & 1
\end{array}\right],
$$


and for transversal Jacobi fields

$$
\mathscr{T}=\left[\begin{array}{cc}
\cos \alpha & 0 \\
\frac{-\sin \alpha}{r \sin \varphi} & \frac{1}{\cos \alpha}
\end{array}\right]\left[\begin{array}{cc}
1 & \frac{l}{\cos \alpha} \\
0 & 1
\end{array}\right]\left[\begin{array}{cc}
\frac{1}{\cos \alpha} & 0 \\
\frac{-\sin \alpha}{r \sin \varphi} & \cos \alpha
\end{array}\right]\left[\begin{array}{cc}
1 & l \\
0 & 1
\end{array}\right] .
$$

Our construction is such that the planar (transversal) Jacobi fields become transversal (planar) in the next face of the box. Hence the evolution of Jacobi fields around the whole periodic orbit is given by the block diagonal matrix

$$
\left[\begin{array}{ll}
\mathscr{T} \mathscr{P} \mathscr{T} \mathscr{P} & \\
& \mathscr{P} \mathscr{T} \mathscr{P} \mathscr{T}
\end{array}\right] .
$$

Clearly the eigenvalues of the two diagonal blocks coincide so that it remains to show that in a certain range of the angle $\alpha$ the matrix $\mathscr{T} \mathscr{P}$ has eigenvalues on the unit circle. It amounts to showing that the trace of $\mathscr{T} \mathscr{P}$ is between -2 and 2 . Alas the expression for the trace of $\mathscr{T} \mathscr{P}$ is quite complicated and its straightforward analysis is more than we can or wish to do. Instead we will show that as $\alpha$ varies from 0 to $\pi /(2 n-1)$ the trace of $\mathscr{T P}$ changes from a value bigger than 2 to a negative value. By continuity there must be an interval for $\alpha$, where the trace of $\mathscr{T} \mathscr{P}$ is between 0 and 2 .

The periodic orbit constructed so far is resonant (the eigenvalues of $\mathscr{T} \mathscr{P}$ and $\mathscr{P} \mathscr{T}$ are the same). We can easily destroy the resonance by taking different $\alpha$ in consecutive faces of the box.

\section{Analytic Details}

We introduce a new parameter

We have then

$$
v=\frac{l \sin \alpha}{r \sin \varphi}
$$

$$
\begin{aligned}
\mathscr{T} & =\left[\begin{array}{cc}
\cos \alpha & l \\
-\frac{v}{l} & \frac{1-v}{\cos \alpha}
\end{array}\right]\left[\begin{array}{cc}
\frac{1}{\cos \alpha} & \frac{l}{\cos \alpha} \\
-\frac{v}{l} & \cos \alpha-v
\end{array}\right] \\
& =\left[\begin{array}{cc}
1-v & l(1+\cos \alpha-v) \\
-\frac{v(2-v)}{l \cos \alpha} & \frac{v^{2}}{\cos \alpha}-\left(1+\frac{2}{\cos \alpha}\right) v+1
\end{array}\right] .
\end{aligned}
$$

Also

$$
\mathscr{P}=\left[\begin{array}{cc}
1+\frac{2 n \tilde{l}}{r \cos \varphi} & l+\tilde{l}+\frac{2 n \tilde{l}}{r \cos \varphi} \\
\frac{4 n}{r \cos \varphi}+\frac{4 n^{2} \tilde{l}}{r^{2} \cos ^{2} \varphi} & 1+\frac{4 n l}{r \cos \varphi}+\frac{2 n \tilde{l}}{r \cos \varphi}+\frac{4 n^{2} \tilde{l}}{r^{2} \cos ^{2} \varphi}
\end{array}\right]
$$


Thus the trace of $\mathscr{T} \mathscr{P}$ is equal to the following unsightly expression:

$$
\begin{aligned}
& \frac{1}{\cos \alpha}(v-\cos \alpha)(v-(2+\cos \alpha))\left(1+\frac{4 n l}{r \cos \varphi}+\frac{2 n \tilde{l}}{r \cos \varphi}+\frac{4 n^{2} \tilde{l}}{r^{2} \cos ^{2} \varphi}\right) \\
& -\cos \alpha\left(1+\frac{2 n \tilde{l}}{r \cos \varphi}\right)-\frac{v(2-v)}{\cos \alpha}\left(1+\frac{\tilde{l}}{l}+\frac{2 n \tilde{l}}{r \cos \varphi}\right) .
\end{aligned}
$$

It is nevertheless easy to see that this expression is negative for $\cos \alpha \leqq v \leqq 2$ and that it exceeds 2 for $v=0$. Hence in some interval of values of $v$ we get the trace between 0 and 2 .

To make $v=\cos \alpha$ we have to have

$$
l=\frac{r \sin \varphi}{\tan \alpha}
$$

$r \sin \varphi / \tan \alpha$ attains all the values from

$$
\frac{r \sin \varphi_{\min }}{\tan \alpha_{\max }}
$$

to $+\infty$, where $\alpha_{\max }=\pi /(2 n-1), \varphi_{\min }=((2 n-3) \pi) / 2(2 n-1)$. Hence we need to require that

$$
l>\frac{r \sin \varphi_{\min }}{\tan \alpha_{\max }} .
$$

If we want to make sure that the semispheres used in the construction do not intersect as $v$ changes from 0 to $\cos \alpha$ we have to satisfy the following inequality (see (5))

$$
\left[r \sin \varphi\left(1+\frac{2}{\cos \alpha}\right)-l \tan \alpha\right]^{2}+r^{2} \sin ^{2} \varphi \geqq 4 r^{2} .
$$

It can be rewritten as

$$
\sin ^{2} \varphi\left[\left(\frac{2+\cos \alpha-v}{\cos \alpha}\right)^{2}+1\right]>4
$$

Clearly it will be satisfied if only

$$
\sin ^{2} \varphi_{\min }>\frac{4}{5}
$$

The last inequality holds if $n \geqq 4$ (it does not for $n=2$ or 3 ; one can check then that the semispheres do intersect at least for some values of $\alpha$ ).

Since we want the condition (6) on $l$ to be as weak as possible we take $n=4$ which yields the requirement that

$$
l>\frac{r \sin \frac{5 \pi}{14}}{\tan \frac{\pi}{7}} \cong 1.8709 r .
$$


If we allow the semispheres to intersect we can put even $n=2$, and then it is sufficient that $l>0.2887 r$.

\section{References}

[A] Arnold, V. I.: Mathematical methods of classical mechanics. Appendix 8. Berlin, Heidelberg, New York: Springer 1978

[B1] Bunimovich, L. A.: On the ergodic properties of nowhere dispersing billiards. Commun. Math. Phys. 65, 295-312 (1979)

[B2] Bunimovich, L. A.: On stochastic dynamics of rays in resonators. Radiofizika 28, 1601-1602 (1985)

[B3] Bunimovich, L. A.: Many-dimensional nowhere despersing billiards with chaotic behavior. Physica D 33, 58-64 (1988)

[B4] Bunimovich, L. A.: Private communication

[D] Donnay, V. J.: Convex billiards with positive entropy (in preparation)

[D-L] Donnay, V. J., Liverani, C.: Ergodic properties of particle motion in potential fields (in preparation)

[K] Knauf, A.: Ergodic and topological properties of Coulombic periodic potentials. Commun. Math. Phys. 110, 89-112 (1987)

[M] Markarian, R.: Billiards with Pesin region of measure one. Commun. Math. Phys. 118, 87-97 (1988)

[S] Sinai, Ya. G.: Dynamical systems with elastic reflections. Russ. Math. Surveys 25, 137-189(1970)

[C-S] Chernov, N. I., Sinai, Ya. G.: Ergodic properties of some systems of 2-dimensional discs and 3-dimensional spheres. Russ. Math. Surveys 42, 181-207 (1987)

[W1] Wojtkowski, M. P.: Principles for the design of billiards with nonvanishing Lyapunov exponents. Commun. Math. Phys. 105, 391-414 (1986)

[W2] Wojtkowski, M. P.: Measure theoretic entropy of the system of hard spheres. Erg. Th. Dyn. Syst. 8, 133-153 (1988)

Communicated by J.-P. Eckmann

Received October 5, 1989 
